\section{Biopsychosocial Approach to the Treatment of Obesity: A Retrospective Review}

\author{
Charles Nguyen ${ }^{1}$, Kevin Bera ${ }^{2 *}$, Robert Bota ${ }^{1 *}$ and Ruth Hsu ${ }^{3 *}$ \\ ${ }^{1}$ Department of Psychiatry, Irvine Medical Center, University of California, \\ Orange, USA \\ ${ }^{2}$ University of Southern California, Los Angeles, USA \\ ${ }^{3}$ University of Stanford, Stanford, USA
}

\begin{abstract}
Background: The increasing prevalence of obesity continues to become a major public health concern, increasing the need for more effective approaches towards weight loss. Current weight loss approaches include weight-loss medications along with nutritional education and exercise, behavioral strategies aimed at lifestyle modifications, or nutritionally restrictive dieting. The purpose of our study was to see if a more comprehensive, "Biopsychosocial" approach that integrates the biological, psychological and behavioral issues, would help patients lose weight.
\end{abstract}

Methods: A "Biopsychosocial" approach was used to help patients lose weight at an outpatient weight loss clinic. The program teaches the role of insulin in weight loss, uses appetite suppressants to reduce cravings, implements cognitive behavioral therapy to reframe thinking, and utilizes behavioral therapy to break old eating habits through six visits. From 2011-2013, 142 patients who completed at least 8 weeks of treatment were retrospectively reviewed and analyzed. Data included demographics, duration of treatment, medications, weight, and BMI.

Results: Participants lost an average of 22.8 pounds, or $10.8 \%$ from the initial weight $(p<0.001)$ over an average of 86 days of treatment.

Conclusion: A "Biopsychosocial" approach is effective for weight loss. Further prospective study is needed.

*Corresponding authors: Kevin Bera, University of Southern California, Los Angeles, USA, Tel: +1 9499330380; E-mail: kevinbera24@gmail.com

Robert Bota, Department of Psychiatry, Irvine Medical Center, University of California, Orange, USA, Tel: +1 7144565951; E-mail: rbota@uci.edu

Ruth Hsu, University of Stanford, Stanford, USA, Tel: +1 6504509523; E-mail: hsur@stanford.edu

Citation: Nguyen C, Bera K, Bota R, Hsu R (2016) Biopsychosocial Approach to the Treatment of Obesity: A Retrospective Review. J Obes Weight Loss : 005

Received: January 16, 2016; Accepted: March 18, 2016; Published: March 30, 2016

\section{Introduction}

The increasing prevalence of obesity in the past several decades has become a major public health concern. More than one-third $(34.9 \%$ or 78.6 million) of US adults are obese [1], with estimated annual medical cost of obesity reaching $\$ 147$ billion dollars in 2008 [2]. In light of this epidemic, the weight loss market has boomed to a $\$ 60$ billion industry [3]. However, effective approaches for weight loss continue to elude us. Current weight loss treatments include prescription weight-loss medications along with nutritional education and exercise, behavioral strategies aimed at lifestyle modifications, or macronutrient-focused diets. While each may be effective initially, studies have found the majority of people were not able to sustain weight loss or health benefits [4].

Anti-obesity medications first appeared in the 1920s when the effects of thyroid hormone were noticed to decrease weight however that practice changed now [5]. Amphetamines and related compounds soon became more common, but complications resulted in stricter FDA oversight [6]. Although helpful with initial weight loss in some, many patients find they do not lose any weight though this method alone [7]. Pharmacotherapy should be used only for those with BMI $>30$ and in combination with lifestyle modifications and other behavioral interventions [8].

Behavioral treatment of obesity first developed based on the belief that obesity was the result of maladaptive eating and exercise habits, which could be corrected by the application of learning principles [9]. While effective in helping modify behavior toward healthier habits, multiple factors such as genetics, metabolic, and hormonal influences are acknowledged toward obesity. Behavioral treatment is based primarily on principles of classical conditioning, which states cues are often linked to food intake [9]. Patients are taught to identify these cues and learn new responses to them. In studies on lifestyle modification, researchers found initial weight loss of $\sim 10 \%$. However, long-term weight loss was not sustained, with weight gain after the first 6 months and continued weight gain with the termination of maintenance therapy [10].

There are numerous diets focusing on macronutrient proportions (low fat, low carbohydrate), with the belief that the right ratio of foods will produce weight loss. However, multiple randomized trials comparing different diets have shown minimal differences in weight loss (mean difference of $<1 \mathrm{~kg}$ ) and metabolic risk factors [11]. Four meta-analyses of diet comparison studies summarizing 13 to 24 clinical trials showed that the only consistent finding among the trials is that adherence was most strongly associated with weight loss [12-15]. Dietary content is only one of many factors influencing adherence but the assumption that one diet is optimal for all persons is unrealistic. Pagoto and Appelhans suggested that advancing obesity treatment requires emphasis on the biological, behavioral, and environmental factors influencing adherence to lifestyle changes [11]. Three large long-term trials have shown that the effects of lifestyle intervention on diabetes prevention are sustained years after the intervention ends [16-18]. Specifically, all three studies involved a highly trained staff to deliver interventions involving dietary and exercise counseling and behavioral modifications. 
In our approach, we address weight loss in multiple areas rather than focusing on one or two aspects. By applying the mental health "Biopsychosocial" model to weight loss to address the biological role of insulin in weight loss [19], psychological approach to change their thinking, and behavioral technique to break their bad habits, we hypothesized that this practical approach is effective to help patients lose weight.

\section{Methods}

A retrospective review of charts of patients ages 18-75, enrolled in a weight loss program at an outpatient clinic from April 1, 2011 until January 1,2013. Patients were included in the sample if they stay on a minimum of 56 days on the program and were excluded if they stayed on more than 140 days. Since cognitive behavioral therapy was used, it's important for patients to participate in at least 8 weeks of treatment in order to have a psychotherapeutic effect. The objective of this short-term study was to measure the weight loss phase so a maximum date of 20 weeks was chosen. The analyzed data included patient demographics, duration of treatment, medications, weight and BMI.

The patients presented for an initial appointment, a 1-week follow-up, and monthly visits thereafter. The initial visit lasted 45 minutes, and each subsequent visit was 10-15 minutes.

At the initial visit, patients were taught about the biopsychosocial method to weight loss (see below) and prescribed a weight loss drug by the same board-certified psychiatrist throughout the visits. At subsequent visits, patients had brief CBT sessions to address cognition distortions, behavioral therapy to break bad habits, nutritional education to lower carbohydrate and sugar intake, and med management of any potential side effects.

The Biopsychosocial program combines the medical knowledge of insulin with cognitive behavioral therapy to reframe thinking and behavioral therapy to break unhealthy habits while forming effective ones to replace them.

\section{Biological: Teaches the role of insulin in weight loss}

Patients were taught about insulin, its role in fat storage, and the importance of avoiding foods that could spike their insulin during the weight loss phase. Patients were taught 4 simple food groups that affect insulin: sweets (desserts, sugary drinks, candy), grains (bread, oatmeal, cereal, rice, and pasta), starchy vegetables (potatoes, corns, carrots, and beets) and high Glycemic Index (GI) fruits. These foods are to be avoided during the weight loss phase to keep insulin low.

Phentermine was used to assist patients with their new eating behavior for most of the patients. Through its mechanisms as a sympathomimetic amine, it increases nor-epinephrine and epinephrine release to help suppress appetite while maintaining energy and alertness.

The study offered a multi-faceted approach, and it was emphasized to the patients that the medications alone do not cause weight loss or affect insulin level. However, they were used as a tool to help fight hunger and energy loss as a result of sudden changes to dietary intake. Patients received either Phentermine $30 \mathrm{mg}$ or $37.5 \mathrm{mg}$ and/or topiramate $25 \mathrm{mg}$ or $50 \mathrm{mg}$, with the majority of patients $(\mathrm{n}=128)$ receiving phentermine $37.5 \mathrm{mg}$ (Table 1 ).

\begin{tabular}{|c|c|c|}
\hline \multicolumn{3}{|c|}{ Medication Usage of Patient Enrollees (N=142) } \\
\hline & $\mathrm{N}$ & Percent (\%) \\
\hline Phentermine $30 \mathrm{mg}$ & 5 & $4 \%$ \\
\hline Phentermine $37.5 \mathrm{mg}$ & 128 & $90.1 \%$ \\
\hline Topiramate $25 \mathrm{mg}$ & 7 & $4.9 \%$ \\
\hline Topiramate $50 \mathrm{mg}$ & 2 & $1.4 \%$ \\
\hline Combination Medication Therapy & 3 & $2.1 \%$ \\
\hline No Medication & 1 & $0.7 \%$ \\
\hline
\end{tabular}

Table 1: Medication Usage of Patient Enrollees Nguyen, Charles 2013.

\section{Psychological: Challenge cognitive distortions and reframe their thinking}

CBT techniques were incorporated into this program to help participants reframe their thinking toward weight loss and dieting. The following phrases were cornerstones to this approach.

For example, the concept of "Thinsulin"- Think thin, think insulin was introduced. Patients reframed their thinking from "weight loss is about eating less" to "weight loss is about lowering insulin". Patients are taught to categorize what they eat into 4 food groups (sweets, grains, vegetables, and fruits). Among the four groups, the patients are taught what kind of foods would spike their insulin level. They were encouraged to eat more protein such as lentils, egg whites, and meat as these foods don't spike insulin level.

"I am not on a diet", the term diet is often associated with deprivation and hunger. Dieting also typically triggers a 'feast-famine' mentality and behavior that leads to inadequate results and discouragement. By changing thought patterns related to diet and nutrition where "on a diet" is replaced with "insulin level mindfulness", a paradigm shift occurs.

"I eat for enjoyment", patients are taught to reframe "I enjoy eating" to "I eat for enjoyment". It's important to acknowledge the importance of enjoyment of life and certainly foods but not whenever you want. By switching the order of two words around, the patients understand that they can have their "enjoyment" (or anything that increases their insulin) only after they eat their breakfast, lunch and dinner.

\section{Behavioral: To break old eating habits and develop new ones}

Patients were instructed to avoid sweets and artificial sweeteners because artificial sweeteners encourage sugar cravings and sugar dependence. To extinguish sugar cravings, patients were asked to avoid all sweets such as desserts, candy, soda, and refrain from adding or consuming any products that contain artificial sweeteners.

Patients were instructed to eat five meals a day to avoid feelings of deprivation and told to never starve themselves. An outline of a day's meal is as follows:

- Breakfast- 1 portion of protein or dairy product

- Morning Snack- 1 portion of low GI fruit (apple, citrus, grapes, berries)

- Lunch- 1 portion of protein and vegetables

- Afternoon Snack- 1 portion of nuts

- Dinner- 1 portion of protein and vegetables

The "Biopsychosocial" approach is a systematic way to change a patient's thinking (psychosocial) in order to apply instruction provided to the patient (e.g., nutrition and insulin education, glucose 
level maintenance) without resorting to chicanery or deprivation, by changing their eating habits (behavior).

\section{Results}

Of 448 patients screened, 142 patients met the inclusion criteria. 310 patients $(69.2 \%)$ of completed at least 4 weeks; $253(56.5 \%)$ patients completed at least 8 weeks, but only $142(31.7 \%)$ of the patient stayed on treatment for the entire duration of 20 weeks. Of the 142 patients, $116(82 \%)$ were female, 44 (31\%) were white, 87 (61.3\%) were Hispanic, and the rest were categorized as "other". The mean age for patients was 40 years old $(\mathrm{SD}=11.31)$ (Table 2).

\begin{tabular}{|c|c|c|}
\hline \multicolumn{3}{|c|}{ Demographics of Patient Enrollees (N=142) } \\
\hline & $\mathrm{N}$ & Percent (\%) \\
\hline Female & 116 & $82 \%$ \\
\hline Male & 26 & $18 \%$ \\
\hline White & 44 & $31.0 \%$ \\
\hline Hispanic & 87 & $61.3 \%$ \\
\hline Other & 11 & $7.7 \%$ \\
\hline \multicolumn{2}{|c|}{ Age } & Mean \\
\hline Number of Days in Program & 40.03 & 11.31 \\
\hline Table 2: Demographics of Patient Enrollees Nguyen, Charles 2013.
\end{tabular}

The primary outcome for the study was a weight change from baseline. A student's t-test was used to compare subjects' weight before and after intervention. There was an average $10.8 \%$ decrease in weight (from average $210 \mathrm{lb}$ to $187 \mathrm{lb}$ ) from baseline to completing the study $((\mathrm{p}<0.001)$ over 86 days. Over the same period of time the BMI decreased from 34.61 to 30.08 ( $\mathrm{p}<0.001) .130(91.5 \%)$ of the patients in the analysis lost at least $5 \%$ of their weight. No patients dropped out in the analysis due to adverse events (Figure 1).

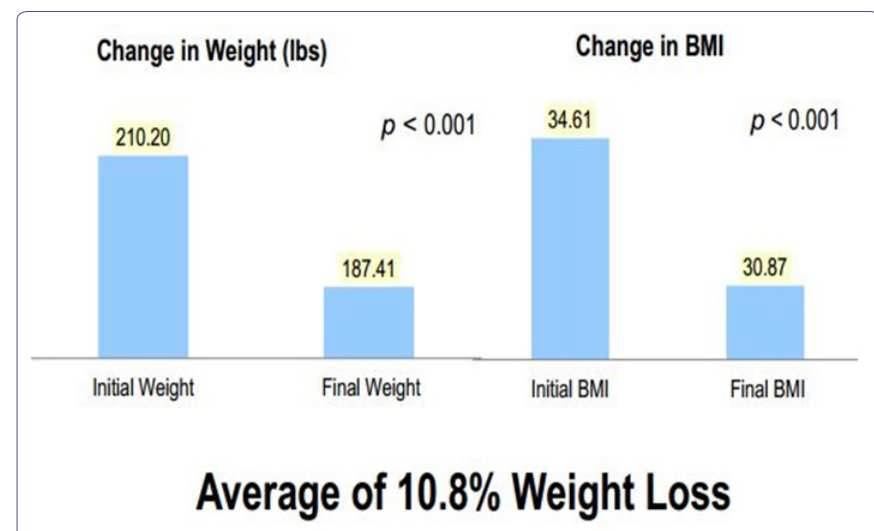

Figure 1: Change in weight and BMI from final to initial weight and BMI measurement over 86 days (2013).

\section{Discussion}

Biopsychosocial approach did allow the clinicians to shift the focus from biomedical model to a more comprehensive approach. Biomedical model focuses on pathophysiology and other biological approaches to a condition such as any underlying deviation from the normal function thus fostering potential dualism. Biopsychosocial approach looks at the person as a whole, from the subatomic level to society and environment the person lives. The interplay between biological, psychological and social factors will change over time and is important to continuously evaluate and incorporate in the treatment plan. The previous studies looking at interventions on one or two approaches provide an initial response but in time fail probably because of the constant dynamic and interplay between those domains.

Cognitive Behavioral Therapy (CBT) is a structured, short-term, present-oriented psychotherapy directed toward solving current problems, and modifying dysfunctional thinking and behavior [20]. CBT has been found useful in weight loss programs, with studies showing increased weight loss when CBT is combined with diet and exercise interventions when compared with diet and exercise alone [21].

In this study the patients were instructed to avoid sweets and artificial sweeteners. Experiments generally have found that sweet taste, whether delivered by sugar or artificial sweeteners, enhanced human appetite [22]. The majority of weight loss programs focus solely on one or two aspects, such as diet, weight loss medication, cognitive-behavioral therapy, or behavioral modification programs. To our knowledge, this is the first program that integrates biological, psychological, and behavioral interventions in an outpatient setting. The resulting $10.8 \%$ reduction in weight and BMI over 86 days of treatment shows the potential efficacy of an outpatient-based program for weight loss using a "Biopsychosocial" intervention. The study reports that a combination of pharmacotherapy, nutritional education, cognitive behavioral therapy, and behavioral modification in short 10-15 minute monthly visits may lead to weight loss.

The limitations of this study include that it was a small, retrospective study with no control group, which makes it difficult to determine exactly which aspects of the treatment were most effective for patients. They may have benefitted from the contact with the clinic staff and the accountability therein as much as from the integrated shifts in their thinking and behavior around food. There were large variations in the length of patient participation and so the results may be varied across patients. Patients were required to self-fund their participation in the program, which limits the socioeconomic spectrum of patients that were able to participate, may have increased the likelihood for weight loss due to financial incentives, and may have self-selected for a more highly motivated group of participants. Additionally, the study only examined patients that completed at least 8 weeks of the program and was not able to assess whether the weight loss was sustained over time after patients left the outpatient clinic's services.

\section{Conclusion}

These preliminary results are promising, but there's a lot of limitations to a retrospective review. Further research into the efficacy of the "Biopsychosocial" approach to weight loss is needed. A randomized, controlled study with a larger pool of patients would be useful in determining the long-term benefits and the widespread applicability of the "Biopsychosocial" approach.

\section{References}

1. Ogden CL, Carroll MD, Kit BK, Flegal KM (2014) Prevalence of childhood and adult obesity in the United States, 2011-2012. JAMA 311: 806-814.

2. Finkelstein EA, Trogdon JG, Cohen JW, Dietz W (2009) Annual medical spending attributable to obesity: payer-and service-specific estimates. Health Aff (Millwood) 28: 822-831.

3. Marketresearch.com (2014) The US Weight Loss Market: 2014 Status Report \& Forecast. Marketresearch.com, Rockville, MD, USA.

4. Mann T, Tomiyama AJ, Westling E, Lew AM, Samuels B, et al. (2007) Medicare's search for effective obesity treatments: diets are not the answer. Am Psychol 62: 220-233. 
5. Kaufman SC, Gross TP, Kennedy DL (1991) Thyroid hormone use: trends in the United States from 1960 through 1988. Thyroid 1: 285-291.

6. Fernstrom JD, Choi S (2008) The development of tolerance to drugs that suppress food intake. Pharmacol Ther 117: 105-122.

7. [No authors listed] (2006) Keeping weight-loss drugs in perspective. If you're dangerously overweight, diet pills may help--but not without major lifestyle changes. Harv Womens Health Watch 13: 1-3.

8. [No authors listed] (1996) Long-term pharmacotherapy in the management of obesity. National Task Force on the Prevention and Treatment of Obesity. JAMA 276: 1907-1915.

9. Stuart RB (1996) Behavioral control of overeating. 1967. Obes Res 4: 411 417.

10. Wadden TA, Butryn ML, Byrne KJ (2004) Efficacy of lifestyle modification for long-term weight control. Obes Res 12: 151-162.

11. Pagoto SL, Appelhans BM (2013) A call for an end to the diet debates. JAMA 310: 687-688.

12. Ajala O, English P, Pinkney J (2013) Systematic review and meta-analysis of different dietary approaches to the management of type 2 diabetes. Am J Clin Nutr 97: 505-516.

13. Wycherley TP, Moran LJ, Clifton PM, Noakes M, Brinkworth GD (2012) Effects of energy-restricted high-protein, low-fat compared with standard-protein, low-fat diets: a meta-analysis of randomized controlled trials. Am J Clin Nutr 96: 1281-1298.

14. Hu T, Mills KT, Yao L, Demanelis K, Eloustaz M, et al. (2012) Effects of low-carbohydrate diets versus low-fat diets on metabolic risk factors: a meta-analysis of randomized controlled clinical trials. Am J Epidemiol 7: 44-54.
15. Bueno NB, de Melo IS, de Oliveira SL, da Rocha Ataide T (2013) Very-low-carbohydrate ketogenic diet $\mathrm{v}$. low-fat diet for long-term weight loss: a meta-analysis of randomized controlled trials. Br J Nutr 110: 1178-1187.

16. Lindström J, Peltonen M, Eriksson JG, llanne-Parikka P, Aunola S, et al. (2013) Improved lifestyle and decreased diabetes risk over 13 years: longterm follow-up of the randomised Finnish Diabetes Prevention Study (DPS). Diabetologia 56: 284-293.

17. Diabetes Prevention Program Research Group, Knowler WC, Fowler SE Hamman RF, Christophi CA, et al. (2009) 10-year follow-up of diabetes incidence and weight loss in the Diabetes Prevention Program Outcomes Study. Lancet 374: 1677-1686.

18. Li G, Zhang P, Wang J, Gregg EW, Yang W, et al. (2008) The long-term effect of lifestyle interventions to prevent diabetes in the China Da Qing Diabetes Prevention Study: a 20-year follow-up study. Lancet 371: 1783-1789.

19. Boden G, Sargrad K, Homko C, Mozzoli M, Stein TP (2005) Effect of a low-carbohydrate diet on appetite, blood glucose levels, and insulin resistance in obese patients with type 2 diabetes. Ann Intern Med 142: 403-411.

20. Beck JS (2011) Cognitive behavior therapy: Basics and Beyond. (2ndedn), The Guildford Press, New York, USA.

21. Shaw K, O'Rourke P, Del Mar C, Kenardy J (2005) Psychological interventions for overweight or obesity. Cochrane Database Syst Rev 18: CD003818.

22. Yang Q (2010) Gain weight by "going diet?" Artificial sweeteners and the neurobiology of sugar cravings: Neuroscience 2010. Yale J Biol Med 83: 101 108. 